\title{
Hydropersulfides, the new kids in the COPD inflammatory town
}

\author{
Luis Puente-Maestu, 1,2,3 Walther Ivan Girón-Matute ${ }^{1,2}$
}

Hydrogen sulfide $\left(\mathrm{H}_{2} \mathrm{~S}\right)$ is one of several small molecules, formerly known primarily as toxic gases (such as nitric oxide (NO) and carbon monoxide) that over the past 20 years have been shown to be endogenously generated small, signalling molecules. ${ }^{1}{ }^{2} \mathrm{H}_{2} \mathrm{~S}$ has accompanied life since its origin. Its toxicological potential, related to the inhibition of cellular respiration, has been known for centuries. ${ }^{3}$ Indeed, the Permian-Triassic mass extinction event has been linked to this toxicity. $\mathrm{H}_{2} \mathrm{~S}$ has been the cause of mining, industrial and sewage accidents, as well as deaths by the inhalation of gases emanating from volcanic and sulfur-rich water wells.

In spite of this felonious record, as it happens with other primeval redox processes, ancient $\mathrm{H}_{2} \mathrm{~S}$ pathways have evolved to play physiological roles in eukaryotic cells.

The rush of discovery for $\mathrm{H}_{2} \mathrm{~S}$ and its derivative compounds started in the late 1980 s by reports of potential biological roles of $\mathrm{H}_{2} \mathrm{~S}$ in modulatory and signalling pathways. ${ }^{4}$ The associated probable health benefits generated a renewed interest in the study of $\mathrm{H}_{2} \mathrm{~S}$, as reflected in the exponential increase in the number of publications. ${ }^{4}$

$\mathrm{H}_{2} \mathrm{~S}$ is produced from L-cysteine by an array of enzymes such as cystathionine $\beta$-synthase (CBS), cystathionine $\gamma$-lyase (CGL), 3-mercaptopyruvate sulfurtransferase (3MST), cysteine aminotransferase and from D-cysteine by D-amino acid oxidase and 3MST. ${ }^{5}$ The expression of these enzymes varies so their relative importance in $\mathrm{H}_{2} \mathrm{~S}$ production depends on the tissue. ${ }^{6}$ In biological systems, $\mathrm{H}_{2} \mathrm{~S}$ is known to spontaneously react with oxidised thiols species, such as dialkyl disulfides (R-S-S-R) or sulfenic acids (R-SOH), to generate hydropersulfides (R-S-SH) groups in peptides and proteins. ${ }^{6}$ Hydropersulfides species are ubiquitous and highly prevalent in mammals

\footnotetext{
${ }^{1}$ Servicio de Neumología del Hospital Universitario Gregorio Marañón c/ Doctor Ezquerdo, Madrid, Spain ${ }^{2}$ Instituto de Investigación Sanitaria Gregorio Marañón c/ Doctor Ezquerdo, Madrid, Spain

${ }^{3}$ Facultad de Medicina de la Universidad Complutense de Madrid. Plaza Ramón y Cajal, Madrid, Spain
}

Correspondence to Professor Luis Puente-Maestu, Servicio de Neumología del Hospital Universitario Gregorio Marañón c/ Doctor Ezquerdo, 4628007 Madrid, Spain; Ipuente@separ.es

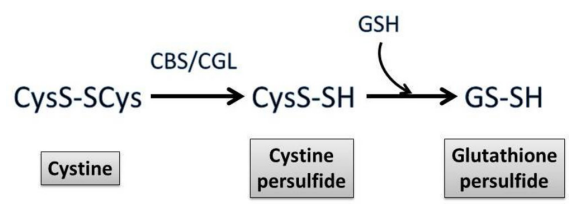

Figure 1 Synthesis of glutathione persulfide. CBS, cystathionine $\beta$-synthase; CGL, cystathionine $\gamma$-lyase; GSH, glutathione.

cells, ${ }^{378}$ apparently protecting proteins from irreversible oxidative damage. ${ }^{6}$ Certain hydropersulfides such as glutathione persulfide (GSSH), cysteine persulfide (CysSSH) and glutathione trisulfide (GSSSH) are highly reactive and extremely powerful antioxidants that actually modulate oxidative stress and regulate redox signalling mediated by various electrophilic oxidants. ${ }^{4}$ Some recent evidence points to enzyme-mediated formation as the most likely source of these species (figure 1$).^{7}$ Of them, the significantly higher GSSH levels detected in cells and tissues indicate that it is likely the principal biological hydropolysulfide. ${ }^{7}$

One important function of the redox system is the regulation of the cell adaptive responses to match the environmental changes. ${ }^{9}$ Indeed, redox signalling is an intricate network that interacts with metalloproteins and cysteine redox switches, thus modulating enzymatic activity and gene transcription by subtle changes in the structure and functional state of proteins. ${ }^{9}$ Reactive oxygen species (ROS) and the powerful nucleophilic by-products resulting from their chemical interaction with NO, known as reactive nitrogen species (RNS), are well-known elements of this redox 'interactome'. ${ }^{1}$ Furthermore, hydropersulfides ${ }^{4}$ are currently recognised as an important direct and indirect redox messenger. ${ }^{110}$

While the signalling function of redox species is intriguing, equally fascinating is the 'dark' role they play in the pathological/degenerative process termed as 'oxidative stress'. ${ }^{11}$ Oxidative stress appears to be a (dys)functional module present in an array of chronic conditions, including hypertension, cardiovascular diseases, obesity, COPD, asthma, diabetes, neurodegenerative diseases and certain forms of cancer, that account for the majority of the global burden of disease. ${ }^{12}$ In the new paradigm for the approach to disease origin and treatment called systems medicine, oxidative stress is an important molecular hub for such set of relevant conditions, ${ }^{3}$ which we could boldly define as redox diseases. ${ }^{13}$

Since the 1990s, oxidative stress, initiated by inhaled oxidants and ROS released from macrophages and neutrophils, has been implicated in the lungs inflammatory response leading to COPD. ${ }^{14} 15$ Either directly or via the formation of lipid peroxidation products, ROS play a role in enhancing inflammation through the activation of stress kinases and redox-sensitive transcription factors, such as nuclear factor-kB and activator protein-1; this results in increased expression of a battery of distinct proinflammatory mediators. ${ }^{14} 15$ RNS also cause lung inflammation, activation of matrix metalloproteinase and inactivation of antiproteases involved in the pathophysiology of the disease. ${ }^{14} 15$ The traditional catalogue of defensive molecules and mechanism to maintain redox balance against the oxidative challenge in lung cells consisted of glutathione and other oxidative radical scavengers such as superoxide dismutase, catalase, glutathione peroxidase and dietary antioxidants. ${ }^{16}$ Nonetheless, the use of selective antioxidants has not, so far, had the anticipated anti-inflammatory effect. This suggests that our current understanding of the underlying pathophysiological redox processes in airways inflammation is incomplete. ${ }^{17}$

In Thorax, Numakura et al report the observations that the amounts of reactive GSSH, CysSSH and GSSSH are decreased in both cultured epithelial cells and fibroblast from the airways as well as in the epithelial lining fluid and sputum obtained from COPD patients who had quit smoking at least 1 year before. ${ }^{18}$ Moreover, the production of reactive hydropersulfides by cultured epithelial cells and fibroblasts was significantly correlated with their presurgery $\mathrm{FEV}_{1}$. By contrast, the synthases (CBS and CGL) were upregulated in the lung tissues and sputum cells from the COPD patients. The study has some limitations.

- It involves a small number of patients and control individuals.

- Participants had lung cancer.

- Differences between ex-smokers, never smokers and ex-smokers with COPD could not be established since the control group was a mix of neversmokers and ex-smokers.

- The measuring techniques are novel; so far the results have not been reproduced and measurements were done in cultured cells from surgical pieces instead of the actual tissue. 
Even so, the findings of Nakamura et al suggest, for the first time, that an abnormal regulation of hydropersulfides species could be a previously unrecognised part of the redox dysbalance, considered as one of the main biological features of COPD inflammation. ${ }^{14} 15$

There is an urgent need to develop more effective therapies for COPD targeting the constitutive inflammatory process. Current therapies with long-acting bronchodilators and inhaled corticosteroids fail to prevent either disease progression or mortality, as they do not suppress the underlying inflammation. The work by Numakura et al heralds a new line of research for a better understanding of the inflammatory process leading to COPD. It also opens the door to new therapeutic approaches by $\mathrm{H}_{2} \mathrm{~S}$-releasing drugs with antioxidant or anti-inflammatory properties. These have already demonstrated considerable promise for the safe treatment of a wide range of inflammatory (redox) disorders and are now in clinical trials for metabolic syndrome, cancer, heart failure and osteoarthritis. ${ }^{2}$

Contributors LP-M is the main author. WIG-M helped with bibliographic search and proofing of the text.

Competing interests None declared.

Provenance and peer review Commissioned; externally peer reviewed.

(c) Article author(s) (or their employer(s) unless otherwise stated in the text of the article) 2017. All rights reserved. No commercial use is permitted unless otherwise expressly granted.

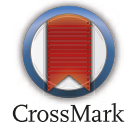

To cite Puente-Maestu L, Girón-Matute WI. Thorax 2017;72:1069-1070.

Published Online First 29 September 2017

\section{SLinked}

http://dx.doi.org/10.1136/thoraxjnl-2016-209359

Thorax 2017;72:1069-1070.

doi:10.1136/thoraxjnl-2017-210703

\section{REFERENCES}

1 Fukuto JM, Carrington SJ, Tantillo DJ, et al. Small molecule signaling agents: the integrated chemistry and biochemistry of nitrogen oxides, oxides of carbon, dioxygen, hydrogen sulfide, and their derived species. Chem Res Toxicol 2012;25:769-93.

2 Wallace JL, Wang R. Hydrogen sulfidebased therapeutics: exploiting a unique but ubiquitous gasotransmitter. Nat Rev Drug Discov 2015; 14:329-45.

3 Cortese-Krott MM, Koning A, Kuhnle GGC, et al. The reactive species interactome: evolutionary emergence, biological significance, and opportunities for redox metabolomics and personalized medicine. Antioxid Redox Signal 2017;27:684-712.

4 Millikin R, Bianco CL, White C, et al. The chemical biology of protein hydropersulfides: studies of a possible protective function of biological hydropersulfide generation. Free Radic Biol Med 2016;97:136-47.

5 Kimura $\mathrm{H}$. The physiological role of hydrogen sulfide and beyond. Nitric Oxide 2014;41:4-10.

6 Cuevasanta E, Möller MN, Alvarez B. Biological chemistry of hydrogen sulfide and persulfides. Arch Biochem Biophys 2017;617:9-25.
7 Ida T, Sawa T, Ihara H, et al. Reactive cysteine persulfides and S-polythiolation regulate oxidative stress and redox signaling. Proc Natl Acad Sci U S A 2014;111:7606-11.

8 Ono K, Akaike T, Sawa T, et al. Redox chemistry and chemical biology of $\mathrm{H} 2 \mathrm{~S}$, hydropersulfides, and derived species: implications of their possible biological activity and utility. Free Radic Biol Med 2014;77:82-94.

9 Jones DP, Sies H. The redox code. Antioxid Redox Signal 2015;23:734-46.

10 Fujii S, Sawa T, Nishida M, et al. Redox signaling regulated by an electrophilic cyclic nucleotide and reactive cysteine persulfides. Arch Biochem Biophys 2016;595:140-6.

11 Jones DP. Redefining oxidative stress. Antioxid Redox Signal 2006;8:1865-79.

12 GBD 2015 Mortality and Causes of Death Collaborators. Global, regional, and national life expectancy, all-cause mortality, and cause-specific mortality for 249 causes of death, 1980-2015: a systematic analysis for the Global Burden of Disease Study 2015. Lancet 2016;388:1459-544.

13 Casas Al, Dao VT, Daiber A, et al. Reactive oxygen-related diseases: therapeutic targets and emerging clinical indications. Antioxid Redox Signal 2015;23:1171-85.

14 Yao H, Rahman I. Current concepts on oxidative/carbonyl stress, inflammation and epigenetics in pathogenesis of chronic obstructive pulmonary disease. Toxicol App/ Pharmacol 2011;254:72-85.

15 MacNee W. Oxidants/antioxidants and COPD. Chest 2000;117(5 Suppl 1):303S-17.

16 Rahman I, Adcock IM. Oxidative stress and redox regulation of lung inflammation in COPD. Eur Respir J 2006;28:219-42.

17 Biswas S, Hwang JW, Kirkham PA, et al. Pharmacological and dietary antioxidant therapies for chronic obstructive pulmonary disease. Curr Med Chem 2013;20:1496-530.

18 Numakura T, Sugiura H, Akaike T, et al. Production of reactive persulfide species in chronic obstructive pulmonary disease. Thorax 2017;72:1074-83. 